\title{
Muon capture on light nuclei
}

\author{
L.E. Marcucci · M. Piarulli
}

Received: date / Accepted: date

\begin{abstract}
This work investigates the muon capture reactions ${ }^{2} \mathrm{H}\left(\mu^{-}, \nu_{\mu}\right) n n$ and ${ }^{3} \mathrm{He}\left(\mu^{-}, \nu \mu\right)^{3} \mathrm{H}$ and the contribution to their total capture rates arising from the axial two-body currents obtained imposing the partially-conserved-axial-current (PCAC) hypothesis. The initial and final $A=2$ and 3 nuclear wave functions are obtained from the Argonne $v_{18}$ two-nucleon potential, in combination with the Urbana IX threenucleon potential in the case of $A=3$. The weak current consists of vector and axial components derived in chiral effective field theory. The low-energy constant entering the vector (axial) component is determined by reproducting the isovector combination of the trinucleon magnetic moment (Gamow-Teller matrix element of tritium betadecay). The total capture rates are 393.1(8) $\mathrm{s}^{-1}$ for $A=2$ and 1488(9) $\mathrm{s}^{-1}$ for $A=3$, where the uncertainties arise from the adopted fitting procedure.
\end{abstract}

Keywords Negative muon capture $\cdot$ Deuteron $\cdot{ }^{3} \mathrm{He} \cdot$ Chiral effective field theory

PACS 23.40.-s $\cdot 21.45 .-\mathrm{v} \cdot 12.39 . \mathrm{Fe}$

\section{Introduction}

There is a significant body of experimental and theoretical work on muon captures in light nuclei, motivated by the fact that the theoretical framework used to study these reactions is the same as that used for weak capture reactions of astrophysical interest, not accessible experimentally. Muon captures, whose rates can be measured, can therefore provide a valuable test of this theoretical framework [1].

"Relativistic Description of Two- and Three-Body Systems in Nuclear Physics", ECT*, October 19-13 2009

L.E. Marcucci

Department of Physics, University of Pisa, and INFN-Pisa,

56127 Pisa, Italy

E-mail: laura.marcucci@edf.unipi.it

M. Piarulli

Department of Physics, Old Dominion University,

Norfolk, VA 23529, USA E-mail: mpiar001@odu.edu 
Very recently [2], the muon capture reactions ${ }^{2} \mathrm{H}\left(\mu^{-}, \nu_{\mu}\right) n n$ and ${ }^{3} \mathrm{He}\left(\mu^{-}, \nu_{\mu}\right)^{3} \mathrm{H}$ have been studied simultaneously in a consistent framework. In particular, the initial and final $A=2$ and 3 nuclear wave functions have been obtained from the Argonne $v_{18}(\mathrm{AV} 18)$ 3] or the chiral $\mathrm{N}^{3} \mathrm{LO}(\mathrm{N} 3 \mathrm{LO})$ 4 two-nucleon potential, in combination with, respectively, the Urbana IX (UIX) [5] or chiral $\mathrm{N}^{2} \mathrm{LO}(\mathrm{N} 2 \mathrm{LO}) \underline{6}$ three-nucleon potentials in the case of $A=3$. The weak current consists of polar- and axial-vector components. The former are related to the isovector piece of the electromagnetic current via the conserved-vector-current (CVC) hypothesis. These and the axial current have been derived within two different frameworks, the standard nuclear physics approach (SNPA), and chiral effective field theory. The first one goes beyond the impulse approximation, by including meson-exchange current contributions and terms arising from the excitation of $\Delta$-isobar degrees of freedom. The second approach includes two-body contributions derived in heavy-baryon chiral perturbation theory within a systematic expansion, up to $\mathrm{N}^{3} \mathrm{LO}[7,8$. To be noticed that, since the transition operator matrix elements are calculated using phenomenological wave functions, it should be viewed as a hybrid chiral effective field theory approach (EFT*). Both SNPA and $\mathrm{EFT}^{*}$ frameworks have been used in studies of weak $p p$ and hep capture reactions in the energy regime relevant to astrophysics $[7,9,10$. The only parameter in the SNPA nuclear weak current model is present in the axial current (the $N$-to- $\Delta$ axial coupling constant) and is determined by fitting the experimental Gamow-Teller matrix element in tritium $\beta$-decay $\left(\mathrm{GT}^{\mathrm{EXP}}\right)$. The SNPA weak vector current, related to the isovector electromagnetic current via CVC, reproduces the trinucleon magnetic moments to better than $1 \%$ 2. In the case of EFT*, three low-energy constants (LECs) appear: one in the axial-vector component, and two in the electromagnetic current. Of these, only one is relevant to the weak vector current, since the other appears in front of an isoscalar operator. The corresponding coupling constants are parameters fixed to reproduce, respectively, $\mathrm{GT}^{\mathrm{EXP}}$ and $A=3$ magnetic moments. To be noticed that the $\mathrm{EFT}^{*}$ currents are obtained performing the Fourier transform from momentum- to coordinate-space with a Gaussian regulator characterized by a cutoff $\Lambda$, varied between 500 and $800 \mathrm{MeV}$. The total capture rates have been found to be $392.0(2.3) \mathrm{s}^{-1}$ for $A=2$ and $1484(13) \mathrm{s}^{-1}$ for $A=3$. The spread accounts for the model dependence, i.e., the dependence on the input Hamiltonian model, the model for the nuclear transition operator, and, in the EFT* calculation, the cutoff sensitivity. This weak model dependence is a consequence of the procedure adopted to constrain the weak current. These results are in very good agreement with the experimental data, in particular with the very accurate measurement of Ref. [1] for the total rate in muon capture on ${ }^{3} \mathrm{He}$.

The muon capture on deuteron has been studied also using the SNPA and the EFT* framework in Ref. [12. The SNPA retains two-body meson-exchange currents derived from the hard pion chiral Lagrangians of the $N \Delta \pi \rho \omega a_{1}$ system and are significantly different from those of Ref. 2]. On the other hand, the EFT* currents are similar to those of Ref. 2], but two differences need to be remarked: (i) the LEC appearing in the axial-vector component $\left(d_{R}\right)$ is not fixed to reproduce $\mathrm{GT}^{\mathrm{EXP}}$, rather the doublet capture rate calculated in SNPA; (ii) a term is added to the leading axial two-body currents, in order to satisfy the partially-conserved-axial-current (PCAC) hypothesis, as constructed in Ref. [13] (called there, and from now on, potential current). The calculated SNPA values for the total capture rate are in the range of $416-430 \mathrm{~s}^{-1}$, depending on the potential model used, resulting in a model dependence much larger than in Ref. 2]. It is also argued that "omitting the potential current causes an enhancement of the doublet transition rate $\Lambda_{1 / 2}$ by $\simeq 1 \%$ " [12]. 
In the present work we repeat the calculation of Ref. 2], in the EFT* approach, adding the potential currents as in Ref. 12]. We restrict our calculation to the AV18 and AV18/UIX potential models, and to a cutoff value of $600 \mathrm{MeV}$, since, as shown in Ref. 2, the dependence on these inputs is less than $1 \%$. We fit the $d_{R}$ coefficient to $\mathrm{GT}^{\mathrm{EXP}}$, and consistently calculate the total rates for muon capture on deuteron and ${ }^{3} \mathrm{He}$. The comparison with the results of Ref. 2] will give and indication of how significant are the potential current contributions for these muon captures.

The paper is organized as follows: in Sec. 2 the theoretical formalism used in the calculation is briefly reviewed. In Sec. 3 the EFT* model for the weak current is described, with the addition of the potential currents. In Sec. 4 the results are presented and discussed, and some concluding remarks are given.

\section{Theoretical formalism}

We briefly review the formalism used in the calculation for the muon capture processes, discussed at length in Refs. 2, 14. The muon capture on deuteron and ${ }^{3} \mathrm{He}$ is induced by the weak interaction Hamiltonian [15], $H_{W}=\frac{G_{V}}{\sqrt{2}} \int \mathrm{d} \mathbf{x} l_{\sigma}(\mathbf{x}) j^{\sigma}(\mathbf{x})$, where $G_{V}$ is

the Fermi coupling constant, $G_{V}=1.14939 \times 10^{-5} \mathrm{GeV}^{-2}$ [16], and $l_{\sigma}$ and $j^{\sigma}$ are the leptonic and hadronic current densities, respectively. The transition amplitude can be written as

$$
\begin{aligned}
& T_{W}\left(f, f_{z} ; s_{1}, s_{2}, h_{\nu}\right) \equiv\left\langle n n, s_{1}, s_{2} ; \nu, h_{\nu}\left|H_{W}\right|(\mu, d) ; f, f_{z}\right\rangle \\
& \quad \simeq \frac{G_{V}}{\sqrt{2}} \psi_{1 s}^{\text {av }} \sum_{s_{\mu} s_{d}}\left\langle\frac{1}{2} s_{\mu}, 1 s_{d} \mid f f_{z}\right\rangle l_{\sigma}\left(h_{\nu}, s_{\mu}\right)\left\langle\Psi_{\mathbf{p}, s_{1} s_{2}}(n n)\left|j^{\sigma}(\mathbf{q})\right| \Psi_{d}\left(s_{d}\right)\right\rangle,
\end{aligned}
$$

for muon capture on deuteron, $\mathbf{p}$ being the $n n$ relative momentum, and 14

$$
\begin{aligned}
& T_{W}\left(f, f_{z} ; s_{3}^{\prime}, h_{\nu}\right) \equiv\left\langle{ }^{3} \mathrm{H}, s_{3}^{\prime} ; \nu, h_{\nu}\left|H_{W}\right|\left(\mu,{ }^{3} \mathrm{He}\right) ; f, f_{z}\right\rangle \\
& \quad \simeq \frac{G_{V}}{\sqrt{2}} \psi_{1 s}^{\text {av }} \sum_{s_{\mu} s_{3}}\left\langle\frac{1}{2} s_{\mu}, \frac{1}{2} s_{3} \mid f f_{z}\right\rangle l_{\sigma}\left(h_{\nu}, s_{\mu}\right)\left\langle\Psi_{3^{3}}\left(s_{3}^{\prime}\right)\left|j^{\sigma}(\mathbf{q})\right| \Psi_{{ }^{3} \mathrm{He}}\left(s_{3}\right)\right\rangle,
\end{aligned}
$$

for muon capture on ${ }^{3} \mathrm{He}$. In order to account for the hyperfine structure in the initial system, the muon and deuteron or ${ }^{3} \mathrm{He}$ spins are coupled to states with total spin $f=1 / 2$ or $3 / 2$ in the deuteron case, and $f=0$ or 1 in the ${ }^{3} \mathrm{He}$ case. In Eqs. (1) and (2) we have defined with $s_{\mu}\left(h_{\nu}\right)$ the muon spin (muon neutrino helicity). The Fourier transform of the nuclear weak current has been introduced as

$$
j^{\sigma}(\mathbf{q})=\int \mathrm{d} \mathbf{x} \mathrm{e}^{\mathrm{i} \mathbf{q} \cdot \mathbf{x}} j^{\sigma}(\mathbf{x}) \equiv(\rho(\mathbf{q}), \mathbf{j}(\mathbf{q}))
$$

with the leptonic momentum transfer $\mathbf{q}$ defined as $\mathbf{q}=\mathbf{k}_{\mu}-\mathbf{k}_{\nu} \simeq-\mathbf{k}_{\nu}, \mathbf{k}_{\mu}$ and $\mathbf{k}_{\nu}$ being the muon and muon neutrino momenta. The function $\psi_{1 s}^{\mathrm{av}}$ has been introduced to take into account the initial bound state of the muon in the atom and the charge distribution of the nucleus. It is typically approximated as 15$]\left|\psi_{1 s}^{\mathrm{av}}\right|^{2}=\frac{\left(\alpha \mu_{\mu d}\right)^{3}}{\pi}$ for muon capture on deuteron, and [14] $\left|\psi_{1 s}^{\text {av }}\right|^{2}=\mathcal{R} \frac{\left(2 \alpha \mu_{\mu^{3} \mathrm{He}}\right)^{3}}{\pi}$ for muon capture on ${ }^{3} \mathrm{He}$, where $\alpha$ is the fine structure constant $(\alpha=1 / 137), \mu_{\mu d}$ and $\mu_{\mu^{3} \mathrm{He}}$ are the reduced masses of the $(\mu, d)$ and $\left(\mu,{ }^{3} \mathrm{He}\right)$ systems, and the factor $\mathcal{R}$ approximately accounts for 
the finite extent of the nuclear charge distribution [15] and is taken to be 0.98 , as in Ref. 2].

In the case of muon capture on deuteron, the final state wave function $\Psi_{\mathbf{p}, s_{1} s_{2}}(n n)$ is expanded in partial waves, and the calculation is restricted to total angular momentum $J \leq 2$ and orbital angular momentum $L \leq 3$, i.e., in a spectroscopic notation, to ${ }^{1} S_{0},{ }^{3} P_{0},{ }^{3} P_{1},{ }^{3} P_{2}-{ }^{3} F_{2}$ and ${ }^{1} D_{2}$. Standard techniques [10, 15] are now used to carry out the multipole expansion of the weak charge, $\rho(\mathbf{q})$, and current, $\mathbf{j}(\mathbf{q})$, operators. Details of the calculation can be found in Ref. 22. Here we only note that all the contributing multipole operators selected by parity and angular momentum selection rules are included, as explained in Ref. 2].

The total capture rate for the two reactions under consideration is then defined as

$$
d \Gamma=2 \pi \delta(\Delta E) \overline{\left|T_{W}\right|^{2}} \times(\text { phase space }),
$$

where $\delta(\Delta E)$ is the energy-conserving $\delta$-function, and the phase space is $d \mathbf{p} d \mathbf{k}_{\nu} /(2 \pi)^{6}$ for muon capture on deuteron and just $d \mathbf{k}_{\nu} /(2 \pi)^{3}$ for muon capture on ${ }^{3} \mathrm{He}$. The following notation has been introduced: (i) for muon capture on deuteron

$$
\overline{\left|T_{W}\right|^{2}}=\frac{1}{2 f+1} \sum_{s_{1} s_{2} h_{\nu}} \sum_{f_{z}}\left|T_{W}\left(f, f_{z} ; s_{1}, s_{2}, h_{\nu}\right)\right|^{2},
$$

and the initial hyperfine state has been fixed to be $f=1 / 2$; (ii) for muon capture on ${ }^{3} \mathrm{He}$

$$
\overline{\left|T_{W}\right|^{2}}=\frac{1}{4} \sum_{s_{3}^{\prime} h_{\nu}} \sum_{f f_{z}}\left|T_{W}\left(f, f_{z} ; s_{3}^{\prime}, h_{\nu}\right)\right|^{2},
$$

and the factor $1 / 4$ follows from assigning the same probability to all different hyperfine states.

After carrying out the spin sums, the differential rate for muon capture on deuteron $\left(d \Gamma^{D} / d p\right)$ and the total rate for muon capture on ${ }^{3} \mathrm{He}\left(\Gamma_{0}\right)$ are easily obtained, and their expressions can be found in Ref. 2. In order to obtain the total rate $\Gamma^{D}$ for muon capture on deuteron, $d \Gamma^{D} / d p$ is plotted versus $p$ and numerically integrated.

Bound and continuum wave functions for both two- and three-nucleon systems entering in Eqs. (11) and (2) are obtained with the hyperspherical-harmonics (HH) expansion method. This method, as implemented in the case of $A=3$ systems, has been reviewed in considerable detail in a series of recent publications 17, 18, We have used the same method in the context of $A=2$ systems, for which of course wave functions could have been obtained by direct solution of the Schrödinger equation. A detailed discussion for the $A=2$ wave functions is given in Ref. 2].

\section{The nuclear weak current operator}

The chiral effective field theory weak current transition operator is taken from Refs. 7 ] and [8], as reviewed in Ref. [2]. It is derived in covariant perturbation theory based on the heavy-baryon formulation of chiral Lagrangians by retaining corrections up to $\mathrm{N}^{3}$ LO. The one-body operators are those listed in Eqs. (17) of Ref. [7] and (4.13)-(4.14) of Ref. 2]. The vector charge and axial current operators retain terms up to $1 / \mathrm{m}^{2}$, while the axial charge and vector current operators retain terms up to $1 / m^{3}, m$ being the nucleon mass. Both $1 / \mathrm{m}^{2}$ and $1 / \mathrm{m}^{3}$ contributions arise when the non-relativistic reduction of the single-nucleon covariant current is pushed to next-to-leading order. The 
two-body vector currents are obtained from the two-body electromagnetic currents via CVC. These are decomposed into four terms $[8]$ : the soft one-pion-exchange $(1 \pi)$ term, vertex corrections to the one-pion exchange $(1 \pi C)$, the two-pion exchange $(2 \pi)$, and a contact-term contribution. Their explicit expressions can be found in Ref. [8]. All the $1 \pi, 1 \pi C$ and $2 \pi$ contributions contain low-energy constants estimated using resonance saturation arguments, and Yukawa functions obtained by performing the Fourier transform from momentum- to coordinate-space with a Gaussian regulator characterized by a cutoff $\Lambda$. Here, as discussed above, we fixed the value of $\Lambda=600 \mathrm{MeV}$. The contact-term electromagnetic contribution is given as sum of two terms, isoscalar and isovector, each one with a coefficient in front $\left(g_{4 S}\right.$ and $\left.g_{4 V}\right)$ fixed to reproduce the experimental values of triton and ${ }^{3} \mathrm{He}$ magnetic moments. For the AV18/UIX Hamiltonian model, with $\Lambda=600 \mathrm{MeV}, g_{4 S}=0.55(1)$ and $g_{4 V}=0.793(6)$, the error being due to numerics [2]. Note that only the isovector contribution is of interest here, but anyway it turns out to be negligible.

The two-body axial current operator consists of two contributions: a one-pion exchange term and a (non-derivative) two-nucleon contact-term. The explicit expression for the contact term can be found in Ref. 7]. Here we review the one-pion exchange term, since we add, in accordance with Ref. 13, the potential current contributions. Therefore, in momentum-space, the one-pion exchange term reads:

$$
\begin{gathered}
\mathbf{j}_{i j}^{\pi}(\mathbf{q} ; A)=\frac{g_{A}}{2 m f_{\pi}^{2}}\left[( \boldsymbol { \tau } _ { i } \times \boldsymbol { \tau } _ { j } ) ^ { a } \left[\frac{\mathrm{i}}{2}\left(1-g_{A}^{2}\right) \frac{\mathbf{p}_{i}+\mathbf{p}_{i}^{\prime}}{2}+\left(\frac{1}{4}+\hat{c}_{4}\right) \boldsymbol{\sigma}_{i} \times \mathbf{k}_{j}\right.\right. \\
\left.+\left(\frac{1+c_{6}+g_{A}^{2}}{4}\right) \boldsymbol{\sigma}_{i} \times \mathbf{q}\right]+2 \hat{c}_{3} \boldsymbol{\tau}_{j}^{a} \mathbf{k}_{j} \\
\left.-\frac{g_{A}^{2}}{4} \boldsymbol{\tau}_{j}^{a}\left(\mathbf{q}+\mathrm{i} \boldsymbol{\sigma}_{i} \times\left(\mathbf{p}_{i}+\mathbf{p}_{i}^{\prime}\right)\right)\right] \frac{\boldsymbol{\sigma}_{j} \cdot \mathbf{k}_{j}}{m_{\pi}^{2}+\mathbf{k}_{j}^{2}}+i \leftrightarrow j .
\end{gathered}
$$

where $\mathbf{k}_{i, j}=\mathbf{p}_{i, j}^{\prime}-\mathbf{p}_{i, j}$, with $\mathbf{p}_{i, j}$ and $\mathbf{p}_{i, j}^{\prime}$ being the initial and final single nucleon momenta, $\mathbf{q}=\mathbf{k}_{i}+\mathbf{k}_{j}, g_{A}=1.2654$ is the axial-vector coupling constant, and $f_{\pi}=93$ $\mathrm{MeV}$ is the pion decay constant. The values used for the coupling constants $\hat{c}_{3}, \hat{c}_{4}$, and $c_{6}$, as obtained from $\pi N$ data, are $\hat{c}_{3}=-3.66, \hat{c}_{4}=2.11$ and $c_{6}=5.83$ [7. The terms proportional to $g_{A}^{2}$ in Eq. (7) are the potential currents. They are the same as in Eq. (21) of Ref. [13] or Eq. (A.17) of Ref. [12. The low-energy constant $d_{R}$, determining the strength of the contact-term two-body axial contribution, has been fixed by reproducing $\mathrm{GT}^{\mathrm{EXP}}$, finding $d_{R}=1.54(8)$. This value should be compared with the corresponding one given in Ref. 2 (see Table V), $d_{R}=1.75(8)$. The difference between these two values of $\simeq 13 \%$ is due to the presence of the potential currents and is comparable with that of Ref. $[12$.

\section{Results}

We present in Table 1 the results for the total rates of muon capture on deuteron, in the doublet hyperfine state $\left(\Gamma^{D}\right)$, and on ${ }^{3} \mathrm{He}\left(\Gamma_{0}\right)$. The deuteron, $n n,{ }^{3} \mathrm{He}$ and ${ }^{3} \mathrm{H}$ wave functions have been calculated with the AV18 3] two- and, when necessary, UIX [5] three-nucleon interactions. The model for the nuclear weak transition operator has been presented in Sec. 3. We compare our results with those of Ref. 2], obtained with the same Hamiltonian model and cutoff $\Lambda$, but without the two-body potential currents elaborated in Ref. [13] and discussed in Sec. 3. Note that here $d_{R}=1.54(8)$, 
Table 1 Total rate for muon capture on deuteron and ${ }^{3} \mathrm{He}$, in $\mathrm{s}^{-1}$. In the $A=2$ case, the different partial wave contributions are indicated. The numbers among parentheses indicate the theoretical uncertainties arising from the adopted fitting procedures. Such uncertainty is not indicated when less than $0.1 \mathrm{~s}^{-1}$. The AV18 and AV18/UIX interactions have been used to calculate the $A=2$ and $A=3$ wave functions. The corresponding results of Ref. 2 are also listed.

\begin{tabular}{lccccccc|l}
\hline & ${ }^{1} S_{0}$ & ${ }^{3} P_{0}$ & ${ }^{3} P_{1}$ & ${ }^{3} P_{2}$ & ${ }^{1} D_{2}$ & ${ }^{3} F_{2}$ & $\Gamma^{D}$ & $\Gamma_{0}$ \\
\hline Present work & $250.1(8)$ & 20.2 & 46.1 & 71.3 & 4.5 & 0.9 & $393.1(8)$ & $1488(9)$ \\
Ref. 2] & $250.0(8)$ & 19.8 & 46.3 & 71.1 & 4.5 & 0.9 & $392.6(8)$ & $1488(9)$ \\
\hline
\end{tabular}

while in Ref. [2] $d_{R}=1.75(8)$. From inspection of the table, we conclude that the two calculations are in remarkable agreement with each other: the differences in $\Gamma_{0}$ and $\Gamma^{D}$ are $\leq 0.1 \%$, well below the theoretical uncertainties. The largest difference, of the order of $2 \%$, is in the ${ }^{3} P_{0}$ partial wave contribution to $\Gamma^{D}$. However, when all the partial wave contributions are summed up, the difference in $\Gamma^{D}$ returns well below the $1 \%$ level.

In conclusion, we have studied the potential currents dictated by PCAC, as elaborated in Refs. 12,13, and we have found that their contributions to the total rates of muon capture on deuteron and ${ }^{3} \mathrm{He}$ are tiny. This result is a consequence of the procedure adopted to constrain the weak current. Finally, we expect that the potential currents will give tiny contributions also in weak capture reactions of astrophysical interest and in those processes whose momentum transfer is small.

\section{References}

1. E.G. Adelberger et al., arXiv:1004.2318

2. L.E. Marcucci et al., arXiv:1008.1172

3. R.B. Wiringa, V.G.J. Stoks, and R. Schiavilla, Phys. Rev. C 51, 38 (1995)

4. D.R. Entem and R. Machleidt, Phys. Rev. C 68, 041001 (2003)

5. B.S. Pudliner et al., Phys. Rev. Lett. 74, 4396 (1995)

6. P. Navrátil, Few-Body Syst. 41, 117 (2007)

7. T.-S. Park et al., Phys. Rev. C 67, 055206 (2003)

8. Y.-H. Song, R. Lazauskas, and T.-S. Park, Phys. Rev. C 79, 064002 (2009)

9. R. Schiavilla et al., Phys. Rev. C 58, 1263 (1998)

10. L.E. Marcucci et al., Phys. Rev. Lett. 84, 5959 (2000); Phys. Rev. C 63, 015801 (2000)

11. P. Ackerbauer et al., Phys. Lett. B 417, 224 (1998)

12. P. Ricci, E. Truhlik, B. Mosconi, and J. Smejkal, Nucl. Phys. A 837, 110 (2010)

13. B. Mosconi, P. Ricci, and E. Truhlík, Eur. Phys. J. A 25, 283 (2005)

14. L.E. Marcucci, R. Schiavilla, A. Kievsky, and M. Viviani, Phys. Rev. C 66, 054003 (2002)

15. J.D. Walecka, Theoretical Nuclear and Subnuclear Physics, Oxford University Press, New York (1995)

16. J.C. Hardy et al., Nucl. Phys. A 509, 429 (1990)

17. A. Kievsky et al., J. Phys. G: Nucl. Part. Phys. 35, 063101 (2008)

18. M. Viviani et al., Few-Body Syst. 39, 159 (2006)

19. L.E Marcucci et al., Phys. Rev. C 80, 034003 (2009) 